\title{
Day-ahead allocation of operation reserve in composite power systems with large-scale centralized wind farms
}

\author{
Jianxue WANG ${ }^{1}$, Ahmed Faheem ZOBAA ${ }^{2}$, Chengchen HUANG ${ }^{1}$, \\ Chen CHEN ${ }^{1}$
}

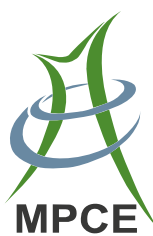

\begin{abstract}
This paper focuses on the day-ahead allocation of operation reserve considering wind power prediction error and network transmission constraints in a composite power system. A two-level model that solves the allocation problem is presented. The upper model allocates operation reserve among subsystems from the economic point of view. In the upper model, transmission constraints of tielines are formulated to represent limited reserve support from the neighboring system due to wind power fluctuation. The lower model evaluates the system on the reserve schedule from the reliability point of view. In the lower model, the reliability evaluation of composite power system is performed by using Monte Carlo simulation in a multi-area system. Wind power prediction errors and tieline constraints are incorporated. The reserve requirements in the upper model are iteratively adjusted by the resulting reliability indices from the lower
\end{abstract}

CrossCheck date: 29 January 2015

Received: 30 September 2014 / Accepted: 29 January 2015 / Published online: 22 September 2015

(C) The Author(s) 2015. This article is published with open access at Springerlink.com

$\triangle$ Jianxue WANG

jxwang@mail.xjtu.edu.cn

Ahmed Faheem ZOBAA

ahmed.zobaa@brunel.ac.uk

Chengchen HUANG

229244524@qq.com

Chen CHEN

yyxfttkx@163.com

1 School of Electrical Engineering, Xi' an Jiaotong University, Xi' an 710049, China

2 College of Engineering, Design and Physical Sciences, Brunel University London, Uxbridge, Middlesex UB8 3PH, UK model. Thus, the reserve allocation is gradually optimized until the system achieves the balance between reliability and economy. A modified two-area reliability test system (RTS) is analyzed to demonstrate the validity of the method.

Keywords Composite system reliability, Reserve allocation, Wind power fluctuation, Operation reserve

\section{Introduction}

As an important renewable energy, wind energy has developed rapidly to meet general requirements on energy saving and emission reduction. However, large-scale integration of wind power greatly affects power systems due to the wind generation characteristics known as randomness and intermittence. These issues have increased research difficulties in many areas, such as power planning, reliability evaluation, and economic dispatch [1-3].

Generally, there are two representative modes for wind farm integration: distributed and centralized connection. In the countries with the distribution disproportion of natural resources like China, the centralized wind farms become the first choice. However, centralization exacerbates the fluctuation of wind power, which may bring about reliability issues as a sudden and severe loss of generation might occur at a high penetration level of wind power. To guarantee the system reliability, additional operation reserve must be prepared for the traditional generation systems. The operation reserve can be categorized as primary, secondary and long-term reserve according to its startup time [4]. In this paper, we concentrate on the secondary reserve (i.e. operation reserves) that the system scheduler allocates among different areas, which is provided by the online units and traditionally used to take 
large variations of load and losses. The objective is to meet high load demand and withstand the impact of wind power fluctuations. Therefore, the magnitude is mostly considered in wind power fluctuation instead of the rate of change.

In a regulated environment like China, the reserve cost could be considered as compensation cost approved by the government. Though operation reserves are not determined by competitive bidding, the system scheduler has to take the responsibility for a compromise between economic issue and reliability issue when considering reserve allocations. Thus for system dispatcher, it is important to determine operation reserves for each area as a tradeoff between economy and reliability.

Relatively generation system reliability models are applied to reserve allocation problems through building the capacity outage probability table (COPT) $[5,6,19]$. In [6], particle swarm optimization (PSO) is used for solving the problem of global reserve dispatch with the assumption that all the lines are reliable. However, the reserve allocation may also handle with the network constraints and reliability concerns to achieve more accurate results. References [7, 8] address the OPF problem embedded with transmission constraints.

The composite system reliability evaluation gives potential shedding load indices in the subsystem [9]. The evaluation is commonly implemented by the methodology of Monte Carlo simulation (MCS). In the conventional works solving reserve allocation problems, however, the composite system reliability evaluation based on MCS has not received direct application since it is difficult to include the process in the optimization frame. In this paper, we proposed a two-level iterative model that includes economic reserve allocation and composite reliability evaluation. Through the iteration method the reserve schedule is progressively optimized, while simultaneously the reliability evaluation based on composite generation and transmission system is completed. Additionally, regional reliability indices are obtained among the iteration steps.

Many large-scale wind farms are sited in the boundary area of local power system. Additionally, the power systems in such areas are composed of sparse electrical networks and insufficient traditional generators. The operation reserve for these wind farms would be provided partially by the transmission corridor which is interconnected with a neighboring system. This means that each local system should schedule some reserve that is used to support neighboring system. However, such support can be abated due to the security constraints of tielines since the tielines must have additional transmission margin to withstand wind power fluctuation, especially under high penetration level.

The security constraint of transmission corridor between the connected systems is specially considered in this paper. The influence of wind power uncertainty on reserve supporting is formulated by the sensitivity factor. The results obtained can provide system operators with explicit information on regional reserve schedule and its corresponding reliability levels.

The realization of reserve allocation includes two key factors: wind uncertainty and network transmission constraints. In the two-level model, the upper model carries out a global scheduling that economically allocates reserve among areas under tieline flow constraints. The lower model evaluates the composite system reliability. MCS is used to incorporate local network constraints and wind power prediction errors. The lower and upper models are linked by reliability and economy under the incumbent reserve schedule.

The paper is organized as follows. Firstly, a two-level model of a multi-area reserve schedule is proposed. Then a composite system reliability evaluation with large-scale wind is discussed. Afterwards, this paper would present and analyze the global reserve allocation model among areas. The solution procedure is given in detail and demonstrated by the IEEE Reliability Test System (RTS). Finally, the conclusion drawn from this study is given.

\section{Two-level framework of multi-area reserve allocation model}

The composite system reliability evaluation usually consists of state sampling, network connectivity analysis and state assessment. The evaluation procedure is complicated, hence it is better to treat this as one independent procedure of economic allocation. The optimization problem can be decomposed into two sub-models as shown in Fig. 1. In the model, the economic reserve allocation and the reliability evaluation are performed iteratively to eventually get an optimized solution which balances the economic and reliability indices.

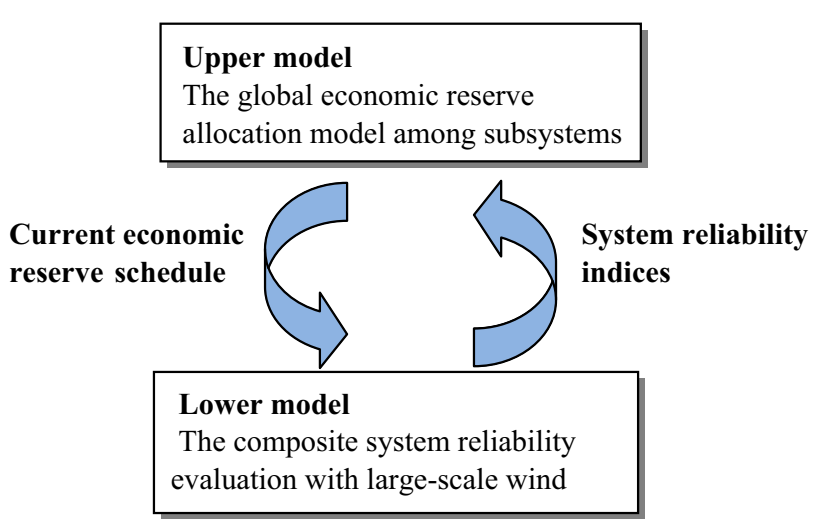

Fig. 1 Two-level model of optimal multi-area reserve schedule 
The upper model minimizes the total reserve cost based on the allocation strategy that is subject to reliability constraints in each area and tieline transmission constraints among different areas.

The lower model evaluates the composite system reliability. The model is utilized to deal with the wind power distribution and local transmission constraint within each area. In this model, loss-of-load probability (LOLP) is used as the reliability index to assess whether the system has adequate reserve [9]. Through comparing the LOLPs of all subsystems, the additional reserve would be allocated to the subsystem with the most urgent need.

The coordinator between the lower model and upper model is the system reliability based on the incumbent reserve schedule. Based on reliability indices from the lower model, the upper model starts from minimum reserve allocation and increases local reserve iteratively. It should be noticed that the increment of local reserve is also a support for the neighboring subsystem when coping with emergency demand. For jointly scheduled reserve in the multi-area system, the increase of local reserve provides convenience for the system operator, but reduces the economy of the whole system. By adjusting the local reserve requirements, the system will balance the reliability and economic level. In practice, the regional power grid corporation is in charge of such a schedule.

\section{Composite system reliability evaluation with large-scale wind by Monte Carlo simulation}

\subsection{Simulation approach of wind fluctuation}

In the sampling of electrical equipment, the two-state model can simulate the operating state and fault state of traditional units. For wind farms, the output can vary from full output to zero in just several minutes.

Although many useful wind speed prediction methods have been discussed and continuously improved [10-12], it is difficult to accurately predict the output of a wind farm in advance due to the obvious stochastic fluctuation of wind resource. This means that the real output of wind farms will deviate to a significant difference from the predicted output. Using the predicted output as the single value of operating state is not accurate in the reliability evaluation, thus the value should be expanded to some distribution. In this paper, wind farm output is simulated using both the forecasted mean value and the sampled deviation. Wind speed correlation has not been taken into consideration.

Wind forecasting error is considered to obey the normal distribution [13, 14]. It can be expressed as:

$$
\left(\frac{P_{w}-P_{w}^{\prime}}{\sigma}\right) \sim N(0,1)
$$

where $P_{w}$ is the real output of wind farm; $P_{w}^{\prime}$ is the forecast output of wind farm; $\sigma$ is the standard deviation. The forecasting accuracy depends on the time horizon $[14,15]$, and a longer time horizon corresponds to a larger $\sigma$.

From (1), the probability distribution of wind output $P_{w}$ can be expressed as:

$P_{w}=P_{w}^{\prime}+\sigma N(0,1)$

For the convenience of calculation, the wind output is represented by several typical discrete states. In contrast to small deviation in the load prediction, wind output remains at obvious variance with the predicted value. For example, the day-ahead predicted wind output should not exceed $20 \%$ deviation in the China standard. Besides the forced outage state, the operating state needs to be subdivided into multiple states. Suppose there are $N_{m}$ discrete states, and $p_{w, m}$ is the state probability of wind output $P_{w, m}$. Referring to the load error distribution [16, 17], the description of wind output distribution is shown in Fig. 2.

The value corresponding to the maximum probability is usually set as the forecasted wind output $P_{w, m}^{\prime}$. Other output values could refer to forms similar to $P_{w, m}^{\prime} \pm \sigma, P_{w, m}^{\prime} \pm 2 \sigma$ and $P_{w, m}^{\prime} \pm 3 \sigma \cdot p_{w, m}$ is further calculated as the cumulative probability on the corresponding range as the shadow zone shown in Fig. 2. After defining $P_{w, m}$ and $p_{w, m}$, a wind farm could be treated as a multi-state generator. Sampling of this multi-state generator is given in Fig. 3, similar to the load sampling [17].

In Fig. 3, $x$ is a random real number in the range $[0,1]$. In the sampling of wind farm, the related wind output is determined by the position of $x$ in the probability range. For example, if $x$ locates in Wind Level $N_{m}-1$, the wind output of this sampling is $P_{w, N_{m}-1}$. The sampling technique of wind farm is used in the evaluation of the composite system reliability, and the discrete state with considerable

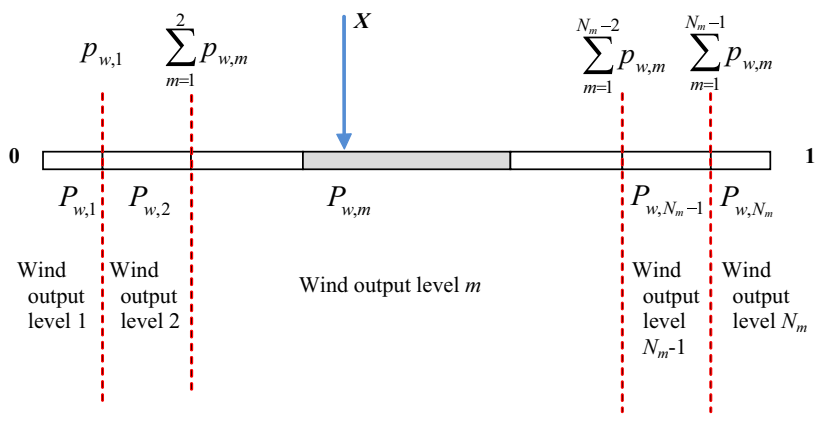

Fig. 2 Discrete distribution of wind forecast error 


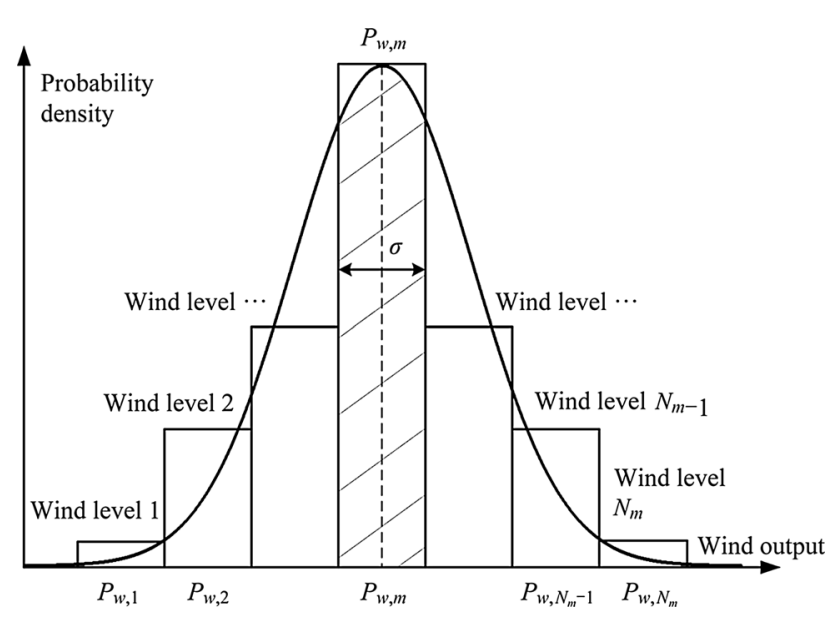

Fig. 3 Probability density of multi-state wind output considering forecast error

variation will affect the power flow in tieline transmission of the global reserve dispatch.

\subsection{Fundamental evaluation of composite system reliability based on Monte Carlo simulation}

The Monte Carlo method can simulate not only single faults, but also various multiple faults, so it is widely used in reliability evaluation of composite power system. Since the intention of the reserve allocation model is to cope with a serious emergency especially during peak load period, this paper used the non-sequential (state sampling) simulation with peak load.

In the sampling stage, the transmission equipment and traditional generators are taken as two-state elements, while the large-scale wind farm with fluctuating output can be conveniently treated as a multi-state element. As the system state in each sampling derives from the initial operating state of electric element, the basic schedule of power flow should be given in advance as the sampling foundation. The analysis of system state requires dealing with many different scenarios, such as whether the system is separate, whether the generation capacity is adequate, whether there is an overload line and how to optimize the load-shedding. Finally, when the convergence criterion of MCS is met, the reliability indices on statistical analysis of all the system states are calculated. The detail of the evaluation process can be found in [18].

\section{Global economic reserve allocation model and discussion}

This section establishes the global economic reserve allocation model referring to [19], which is shown as the upper model in Fig. 1. The objective is to schedule operation reserve of the whole system with minimum cost, as shown in (3).

$\min \left(\sum_{i=1}^{N_{R}} \rho_{i} R_{i}\right)$

where $N_{R}$ is the number of generators to provide operation reserve; $R i$ is the selected reserve of generator $i ; \rho_{i}$ is the reserve cost of generator $i$, which could be the offer price of generator $i$ for reserve service, or the compensation cost for the reserve service according to the system operation rules. For example, in some regional systems of China, if the coal-fired unit is scheduled to provide reserve, the compensation cost for one hour is $¥ 100 / \mathrm{MW}$ (about \$16/ MW), and the reserve from hydro station is much cheaper.

In detail, the allocation problem is subject to some constraints due to reliability requirement and technical limitation.

1) Maximum available reserve of each generator

The technology limitation of reserve is written as:

$0 \leq R_{i} \leq \bar{R}_{i}$

where $\bar{R}_{i}$ is the maximum reserve of generator $i$; $i=1,2, \cdots, N_{R}$. Usually, $\bar{R}_{i}$ is set as maximum capacity $\bar{P}_{\mathrm{g}, i}$ minus scheduled generation capacity $P g, i$.

2) Minimum local reserve requirement of each area

$\sum_{i=1}^{N_{R, l}} R_{i} \geq \underline{D}_{r, l}$

where $l=1,2, \cdots, N_{a} ; N_{a}$ is the number of areas; $N_{R, l}$ is the number of reserve generators in area $l ; \underline{D}_{r, l}$ is the minimum local reserve requirement.

Even if the tieline transmission corridors have plentiful capacity, keeping some local reserve in each subsystem is necessary in order to deal with various emergencies quickly and flexibly. However, redundant local reserve will reduce the economy of the global reserve allocation. In the other words, a large value means some loss of interconnected benefit.

$\underline{D}_{r, l}$ is an important indicator coordinating the upper model with the lower model. In the initial step of the solution procedure, all the $\underline{D}_{r, l}$ are given small values. In such a configuration, the cheapest reserve in the multi-area system will be selected. With an increase of $\underline{D}_{r, l}$ of the subsystem from the least reliable level, the reliability will be enhanced, but the economy will be reduced. Therefore, the adjustment process of $\underline{D}_{r, l}$ is also the process to balance the economy and reliability.

3) System and subsystem reliability constraint 
$p_{L O L P, l} \leq \bar{p}_{L O L P, l}$

$p_{L O L P} \leq \bar{p}_{L O L P}$

where $p_{L O L P, l}$ is the reliability index LOLP of the area $l$ based on the incumbent schedule; $\bar{p}_{L O L P, l}$ and $\bar{p}_{L O L P}$ are the required LOLP of area $l$ and the required system LOLP respectively. As a lower LOLP value corresponds to a higher reliability level, the constraint gives the reliability requirement of the subsystem. $\bar{p}_{L O L P, l}$ can use the exact value, like 0.01 [20] or the proportion form, $\bar{p}_{L O L P}=\alpha p_{L O L P, l}^{0}$. For example, $\alpha=10 \%$ means the reserve will reduce $90 \%$ probability of shedding load.

4) Total reserve limits of the whole system

$\underline{D}_{R} \leq \sum_{i=1}^{N_{R}} R_{i} \leq \bar{D}_{R}$

where $\bar{D}_{R}$ is the maximum total reserve requirement of the whole system; $\underline{D}_{R}$ is the minimum total reserve requirement of the whole system. This constraint gives the range for the total selected reserve.

$\underline{D}_{R}$ is usually set as a small value, and $\bar{D}_{R}$ can be determined from the equation

$\bar{D}_{R}=\sum_{l=1}^{N_{a}} \bar{D}_{r, l}$

where $\bar{D}_{r, l}$ is the individual reserve requirement of area $l$ which could be set according to the deterministic reserve rule. These values have several forms, such as certain proportion of the maximum load, the maximum running generator capacity, or the combination of the two forms. In the union for the co-ordination of transmission of electricity (UCTE), the recommended value for up reserve limit is near to $6 \sqrt{P_{d, \text { max }}}$ where $P_{d, \text { max }}$ is the maximum hourly forecasted load [21].

In the worst condition, all the local reserve requirements have reached the individual reserve requirements, which means each subsystem will rarely share the reserve support from other subsystems. In practice, the reserve schedule always provides some mutual support, and hence the sum of a selected reserve is smaller than the sum of the individual requirements. The reserve difference can be seen as one index of the interconnected benefits.

5) Tieline transmission constraint

$-\bar{P}_{T, k} \leq P_{T, k}+\sum_{i=1}^{N_{R}} G_{k-i} R_{i}+\sum_{j=1}^{N_{W}} G_{k-j} \Delta P_{w, j} \leq \bar{P}_{T, k}$

where $k=1,2, \cdots, N_{T} ; N_{T}$ is the number of tielines; $N_{W}$ is the number of wind farms; $P_{T, k}$ is the initial power flow of branch $k ; \bar{P}_{T, k}$ is the maximum transmission limitation of branch $k ; G_{k-i}$ is the generalized generation shift distribution factor (GGDF) of the generator $i$ on branch $k$ [22]; $\Delta P_{w, j}$ is the magnitude of wind fluctuation.

The GGDF is often used in some multi-area economic dispatch [23]. In the proposed allocation problem, the variation of dispatched reserve capacities and wind fluctuation can be included in the tieline transmission constraint by using GGDF.

Although there are many scenarios, only the most serious variations of the two factors, wind fluctuation and transmission constraints, are considered in this tieline constraint. For wind fluctuation, the forecast error of wind output obeys a normal distribution as shown in Fig. 2. The serious wind fluctuation is set as $\Delta P_{w, j}=k \sigma$, where $k=3$, 3.5 or other values [24-26]. Simultaneously, schedulable reserve should not exceed the selected reserve in advance, so the maximum schedulable reserve is set as the selected reserve $R_{i}$.

\section{Build process of multi-area reserve allocation model}

As the model is constructed from the two-level framework, it significantly reduces the solution difficulty. The upper model, global reserve allocation model among areas, is a linear programming model and can be solved by mature algorithms. The lower model, the composite system reliability evaluation, can be executed by MCS. The process is introduced in detail as below.

Step 1: Set the electrical element parameters, including branch, generator, load, etc. Meanwhile, set $\bar{P}_{T, k}, \underline{D}_{r, l}$, $\bar{p}_{L O L P, l}$ and $\rho_{i}$. Initialize the minimum value of local reserve requirement $\underline{\boldsymbol{D}}_{r}^{0}=\left[\underline{D}_{r, 1}^{0}, \underline{D}_{r, 2}^{0}, \cdots, \underline{D}_{r, N_{a}-1}^{0}, \underline{D}_{r, N_{a}}^{0}\right]$. In addition, the forecasted wind output distribution is provided, including forecast point value and the statistical deviation.

Step 2: Suppose $n$ is the iterative number, and its initial value is 1 . Set the incremental capacity $\Delta D$ of local reserve requirement.

Step 3: Calculate the GGDF, i.e., $G_{k-i}$, using the results of the scheduled basic power flow.

Step 4: Solve the global reserve allocation model, obtaining the reserve schedule $\boldsymbol{R}^{n}=\left[R_{1}^{n}, R_{2}^{n}, \cdots, R_{N_{a}-1}^{n}, R_{N_{a}}^{n}\right]$.

Step 5: Judge whether the sum of selected reserve is still less than $\bar{D}_{R}$. If it does not exceed the requirement, go to the next step. Otherwise go to Step10.

Step 6: Use Monte Carlo simulation to evaluate the composite system index $p_{L O L P, l}^{(n)}$ of area $l$ and $p_{L O L P}^{(n)}$ of the overall system based on the incumbent electricity and reserve schedule. 


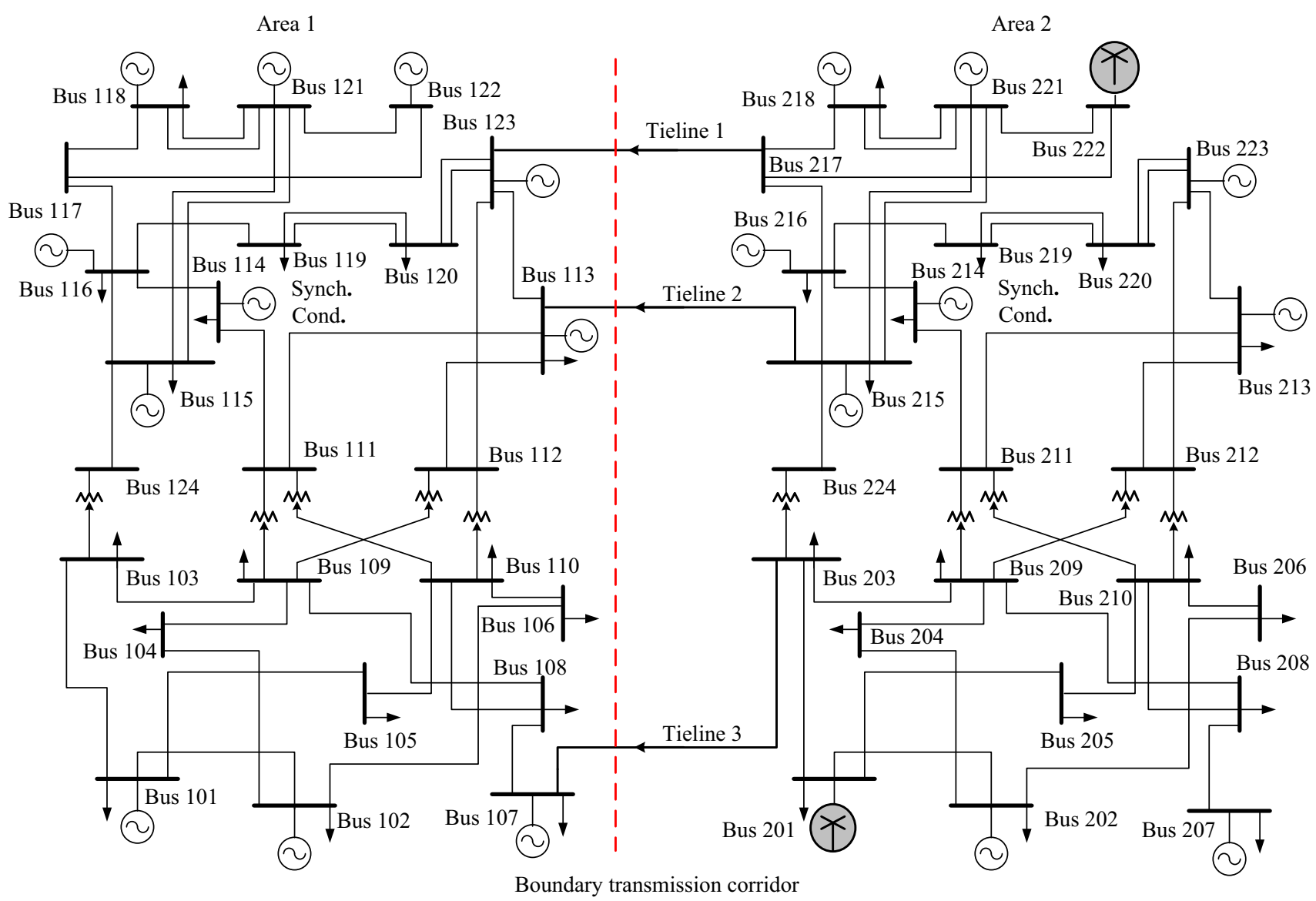

Fig. 4 Modified RTS96 two-area system with wind farms

Table 1 Scheduled output data at each bus

\begin{tabular}{llllll}
\hline Bus & Area & $P(\mathrm{MW})$ & Bus & Area & $P(\mathrm{MW})$ \\
\hline 101 & 1 & 20 & 201 & 2 & 192 \\
102 & 1 & 172 & 202 & 2 & 172 \\
107 & 1 & 228 & 207 & 2 & 228 \\
113 & 1 & 286.1 & 213 & 2 & 285.3 \\
115 & 1 & 215 & 215 & 2 & 215 \\
116 & 1 & 155 & 216 & 2 & 155 \\
118 & 1 & 400 & 218 & 2 & 400 \\
121 & 1 & 400 & 221 & 2 & 400 \\
122 & 1 & 100 & 222 & 2 & 500 \\
123 & 1 & 660 & 223 & 2 & 660 \\
\hline
\end{tabular}

Step 7: Judge whether all the reliability indices of subsystems have reached the given levels. If there is at least one area that does not satisfy the given index, go to Step 8 . Otherwise, go to Step 10.

Step 8: In the $n^{\text {th }}$ loop, calculate the reliability $p_{L O L P, j}^{l,(n)}$ when an incremental capacity $\Delta D$ is added to the local reserve requirement of area $l$. Then calculate the differences $\Delta p_{L O L P, j}^{l}=p_{L O L P, j}^{l,(n)}-p_{L O L P, j}^{l,(n-1)}$ and sum them all, or
Table 2 Tieline data in boundary transmission corridor

\begin{tabular}{llll}
\hline Bus (from) & Bus (to) & $\begin{array}{l}\text { Basic power } \\
\text { flow (MW) }\end{array}$ & $\begin{array}{l}\text { Maximum transmission } \\
\text { capacity (MW) }\end{array}$ \\
\hline 217 & 123 & 309.071 & 625 \\
215 & 113 & 411.969 & 625 \\
203 & 107 & 114.413 & 220 \\
\hline
\end{tabular}

simply calculate the difference of the whole system reliability.

Step 9: Pick the area $l$ with maximum change of reserve requirement and set $\underline{\boldsymbol{D}}_{r}^{n+1}=\left[\underline{D}_{r, 1}^{n}, \underline{D}_{r, 2}^{n}, \cdots, \underline{D}_{r, l}^{n}+\Delta D, \ldots, \underline{D}_{r, N_{a}-1}^{n}, \underline{D}_{r, N_{a}}^{n}\right]$. Let $n=n+1$, then return to Step 4 .

Step 10: Print the final multi-area reserve schedule.

The solution procedure has two terminal conditions in Step 5 and 7. When all the subsystems satisfy the required reliability level, or the total selected capacity exceeds the reserve requirement, the iteration will stop and finish the reserve schedule. Obviously, the solution we need is the result with the first terminal condition. 
Table 3 Reserve cost data

\begin{tabular}{lllllrl}
\hline Unit & Bus & Number & Area & Fuel & $\begin{array}{l}\text { Reserve } \\
(\mathrm{MW})\end{array}$ & $\begin{array}{l}\text { Reserve cost } \\
(\$ / \mathrm{MW})\end{array}$ \\
\hline 20 & 101,102 & 4 & 1 & $\# 2$ oil & 10.00 & 33 \\
100 & 107 & 3 & 1 & $\# 6$ oil & 24.00 & 19.8 \\
197 & 113 & 3 & 1 & $\# 6$ oil & 101.68 & 22 \\
$\mathrm{t} 20$ & 202 & 2 & 2 & $\# 2$ oil & 10.00 & 30 \\
100 & 207 & 3 & 2 & $\# 6$ oil & 24.00 & 18 \\
197 & 213 & 3 & 2 & $\# 6$ oil & 101.90 & 20 \\
\hline
\end{tabular}

\section{Case study}

\subsection{Test system}

The modified two-area system of IEEE RTS96 is employed in the case study, which is connected by three tielines as shown in Fig. 4.

The RTS has been modified to highlight the large-scale wind integration and mutual reserve support. The basic data of the two-area RTS96 in peak load can be found in [27]. Here we only provide the modified data.

1) In aspect of generator, two large-scale wind farms are added into the system. Wind farm 1 at Bus 201 has 250 MW installed capacity. Wind farm 2 at Bus 222 has 600 MW installed capacity. Meanwhile, remove two 76 MW units from Bus 101, four 50 MW units from Bus 102, all units from Bus 201, and all units from Bus 222. The results of modified units are: two 20 MW units at Bus 101, two $50 \mathrm{MW}$ units at Bus 122, one $250 \mathrm{MW}$ wind farm at Bus 201, and one $600 \mathrm{MW}$ wind farm at Bus 222.

2) In aspect of load, halve the load on Bus 202, 207, 213, 215, 218. Meanwhile, increase each load on Bus 102, $107,113,115,118$ by $50 \%$. Consequently, the total load is still 5700 MW. Subsystem of Area 1 is the importing area

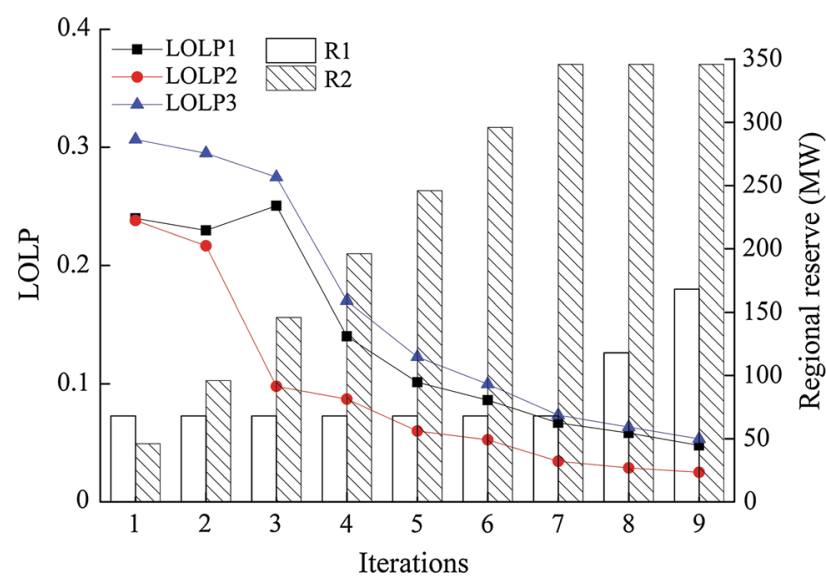

Fig. 5 Iteration process of reserve allocation
Table 4 Reserve allocated to each unit

\begin{tabular}{lll}
\hline Unit & Bus & Reserve (MW) \\
\hline 100 & 107 & 24 \\
100 & 107 & 24 \\
100 & 107 & 24 \\
197 & 113 & 96 \\
100 & 207 & 24 \\
100 & 207 & 24 \\
100 & 207 & 24 \\
197 & 213 & 70.2 \\
197 & 213 & 101.9 \\
197 & 213 & 101.9 \\
\hline
\end{tabular}

with 3418.5 MW load and 3053.0 MW generator capacity. Subsystem of Area 2 is the exporting area with $2281.5 \mathrm{MW}$ load and 3763.0 MW generator capacity.

The output of the normal units is given in Table 1. Based on the scheduled output, the basic power flow can be calculated.

The tieline data are given in Table 2. Since the loadshedding and the global reserve allocation model are both based on the DC flow, the basic power flow and transmission limitation are given only in active power.

In Table 2, although there are heavy power flows in the tielines, it is still capable for the transmission corridors to take on more of a load.

3) In aspect of equipment reliability, the forced outage rates of all the units and branches have been cut by $50 \%$ to get the more reliable system. The given acceptable LOLPs of each subsystem $p_{L O L P, 1} \leq 0.05, p_{L O L P, 2} \leq 0.05$. The acceptable overall system LOLP is set to 0.06 . The initial value of minimum local reserve requirement is set to $2 \%$ of the maximum local load, i.e., $\underline{D}_{r, 1}=68 \mathrm{MW}$ and $\underline{D}_{r, 2}=46$ MW. The maximum reserve requirement of the whole multi-area system is set as $10 \%$ of the total load, i.e. $\bar{D}_{R}=$ $570 \mathrm{MW}$.

The basic reserve cost is quoted from the data given in [23], and the reserve cost of Area 1 is increased to $110 \%$, as shown in Table 3.

From Table 3, we could find that 18 units provide 814.59 MW reserve to satisfy the maximum 570 MW

Table 5 Reserve allocation results

\begin{tabular}{llll}
\hline Results & Basic case & Without wind farm & Tighter constraint \\
\hline R1 (MW) & 168 & 318 & 416.89 \\
R2 (MW) & 346 & 146 & 46 \\
LOLP1 & 0.0478 & 0.0497 & 0.042 \\
LOLP2 & 0.025 & 0.0499 & 0.0352 \\
Cost (\$) & 10313.60 & 9613.60 & 10281.20 \\
\hline
\end{tabular}


Table 6 Total simulation time and iteration numbers

\begin{tabular}{llll}
\hline$\beta_{\text {LOLP (\%) }}$ & System LOLP & Iteration numbers & CPU-time (min) \\
\hline 3 & 0.0571 & 8 & 14 \\
2 & 0.0544 & 9 & 28 \\
1 & 0.0550 & 9 & 94 \\
\hline
\end{tabular}

requirement. The presented model is to find the rational reserve allocation between the two areas under the acceptable reliability level.

The forecast output of Wind farm 1 is 192 MW and its deviation $\sigma$ is $12.8 \mathrm{MW}$. The forecast output of Wind farm 2 is $500 \mathrm{MW}$ and its deviation $\sigma$ is $33.3 \mathrm{MW}$. In the tieline transmission constraint, a $3 \sigma$ rule is used to express the wind fluctuation.

\subsection{Basic result of reserve allocation}

Figure 5 shows the iteration process of reserve allocation, with an increasing $50 \mathrm{MW}$ capacity in each iteration, where LOLP1 is LOLP of Area 1; LOLP2 is LOLP of Area 2; LOLPs is LOLP of the overall system; R1 is the selected reserve of Area 1; R2 is the selected reserve of Area 2.

Figure 5 shows that the LOLP index gradually decreases with the increase of reserve. At the final iteration, LOLP1 and LOLP2 are both below the given value 0.05 , meanwhile the overall system LOLPs is below 0.06. As the cost of keeping these reliability levels, the system takes $\$ 10,313.6$ to buy $168 \mathrm{MW}$ reserve in Area 1 and $346 \mathrm{MW}$ reserve in Area 2. In the iteration process, there is a rising jump between the 2nd and 3th iteration of the LOLP of Area 1, i.e., the reserve is increasing while the reliability index is not decreasing, which is caused by some deviation of the stochastic sampling in the Monte Carlo method. However, the total LOLP tendency is definitely decreasing.

Table 4 gives the reserve allocated by each unit in detail.

The reserve allocation results in Table 4 show that cheaper reserves in Area 2 are selected for plentiful remaining capacity in the transmission corridor.

\subsection{Analysis of reserve allocation with various wind farms and various transmission constraints}

The analysis of the cases with different wind power is illustrated in Table 5. In the case without wind power, some thermal units of equal capacity are added to replace the wind farms.

The results show that greater reserve needs to be allocated for Area 1 without wind power. When large-scale wind farms are connected to Area 2, it requires more local reserve to smooth the wind fluctuations and reach the given reliability level of Area 2. In order to reach the same reliability level, integrating large-scale wind power needs an additional 50 MW capacity and $\$ 700$ cost for the reserve.

To find the influence of the transmission constraint, we decrease the upper capacity limit of the three tielines from [625 625 220] (MW) to [480 480 150] (MW), and the other data remain the same as in the basic case. The calculation is carried out and the comparison is illustrated as the tighter constraint case in Table 5 .

When the transmission constraint becomes tighter, the reserve allocation would be adjusted. Since Area 1 can only acquire limited support from Area 2 with such a strict constraint on the tielines, Area 1 has to prepare more operation reserve to ensure its reliability. At the same time, Area 2 can share the reserve support of Area 1, so it can keep the minimum local reserve.

\subsection{Analysis of calculation efficiency and precision with different convergence criteria}

The MCS convergence criterion is the coefficient of variance of the LOLP index, noted as $\beta_{\text {LOLP. The definition }}$ of $\beta_{\text {LOLP }}$ can be found in [28]. To discuss the contradiction of precision and efficiency, we test the proposed method on different convergence criteria. The total simulation time and iteration numbers of the basic case are shown in Table 6.

The results show that the calculation converges after only 8 iterations for $3 \%$ threshold. The resulting reserve schedule is different from those for $2 \%$ and $1 \%$. This result illustrates that the convergence is too early and the precision for $3 \%$ threshold is not adequate. The calculation for $2 \%$ threshold has identical iteration numbers to the calculation for $1 \%$ threshold, but has much less simulation time. The difference of system LOLP index when $\beta_{\text {LOLP }}<2$ is very small and the reserve schedules are the same. Thus $\beta_{\text {LOLP }}=2$ is recommended for the proposed system. It should be pointed out that the LOLP index calculated in this methodology is slightly instable, better index should be investigated in the further research.

\section{Conclusions}

An allocation method for operating reserve is investigated in composite power systems with centralized wind farms. In the case study, the modified two-area RTS is employed to provide numerical results of the proposed method. The results show that it is possible to solve this 
complex allocation problem by a heuristic iterative method. Thus an optimized reserve schedule is obtained. Our reserve allocation model is meaningful not only for obtaining an economic solution of reserve allocation, but also for keeping system reliability above a certain level. In the future research, the correlation between wind farms, and interrelation between wind and load will be studied in a more detailed model.

Acknowledgment This work was supported by National Natural Science Foundation of China (No. 51277141) and National High Technology Research and Development Program of China (863 Program) (No. 2011AA05A103).

Open Access This article is distributed under the terms of the Creative Commons Attribution 4.0 International License (http:// creativecommons.org/licenses/by/4.0/), which permits unrestricted use, distribution, and reproduction in any medium, provided you give appropriate credit to the original author(s) and the source, provide a link to the Creative Commons license, and indicate if changes were made.

\section{References}

[1] Ummels BC, Gibescu M, Pelgrum E et al (2007) Impacts of wind power on thermal generation unit commitment and dispatch. IEEE Trans Energy Conver 22(1):44-51

[2] Zhao X, Wu L, Zhang S (2013) Joint environmental and economic power dispatch considering wind power integration: Empirical analysis from Liaoning Province of China. Renew Energy 52:260-265

[3] Shan J, Botterud A, Ryan SM (2014) Temporal versus stochastic granularity in thermal generation capacity planning with wind power. IEEE Trans Power Syst 29(5):2033-2041

[4] Dany G (2001) Power reserve in interconnected systems with high wind power production. In: Proceedings of the 2001 IEEE Porto power tech conference, Vol 4, Porto, 10-13 Sept 2001, 6p

[5] Matos MA, Bessa RJ (2011) Setting the operating reserve using probabilistic wind power forecasts. IEEE Trans Power Syst 26(2):594-603

[6] Hajian-Hoseinabadi H, Fotuhi-Firuzabad M, Hajian M (2008) Optimal allocation of spinning reserve in a restructured power system using particle swarm optimization. In: Proceedings of the Canadian conference on electrical and computer engineering (CCECE'08), Niagara Falls, 4-7 May 2008, pp 535-540

[7] Ahmadi-Khatir A, Conejo AJ, Cherkaoui R (2014) Multi-area unit scheduling and reserve allocation under wind power uncertainty. IEEE Trans Power Syst 29(4):1701-1710

[8] Lescano GA, Aurich MC, Ohishi T(2008) Optimal spinning reserve allocation considering transmission constraints. In: Proceedings of the Power and Energy Society general meetingConversion and delivery of electrical energy in the 21 st century, Pittsburgh, 20-24 Jul 2008, 6 pp

[9] Billinton R, Allan RN (1984) Reliability evaluation of power systems. Pitman Advanced Publishing Program, Boston

[10] Billinton R, Gao Y, Karki R (2009) Composite system adequacy assessment incorporating large-scale wind energy conversion systems considering wind speed correlation. IEEE Trans Power Syst 24(3):1375-1382
[11] Lei M, Shiyan L, Chuanwen J et al (2009) A review on the forecasting of wind speed and generated power. Renew Sustain Energy Rev 13(4):915-920

[12] Foley AM, Leahy PG, Marvuglia A et al (2012) Current methods and advances in forecasting of wind power generation. Renew Energy 37(1):1-8

[13] Makarov YV, Huang Z, Etingov P, et al (2010) Incorporating wind generation and load forecast uncertainties into power grid operations. PNNL-19189, Pacific Northwest National Laboratory, Seattle

[14] Doherty R, O’Malley M (2005) A new approach to quantify reserve demand in systems with significant installed wind capacity. IEEE Trans Power Syst 20(2):587-595

[15] Strbac G, Shakoor A, Black M et al (2007) Impact of wind generation on the operation and development of the UK electricity systems. Electr Power Syst Res 77(9):1214-1227

[16] Bouffard F, Galiana FD (2008) Stochastic security for operations planning with significant wind power generation. In: Proceedings of the Power and Energy Society general meetingConversion and delivery of electrical energy in the 21 st century, Pittsburgh, 20-24 Jul 2008, 11 pp

[17] Amjady N, Aghaei J, Shayanfar HA (2009) Stochastic multiobjective market clearing of joint energy and reserves auctions ensuring power system security. IEEE Trans Power Syst 24(4):1841-1854

[18] Li WY (2014) Risk assessment of power systems: Models, methods, and applications. IEEE Press, Piscataway

[19] Wang JX, Wang XF, Ding XY (2006) Study on zonal reserve model in power market. Proceedings of the CSEE 26(18):28-33

[20] Bessa RJ, Matos MA (2010) Comparison of probabilistic and deterministic approaches for setting operating reserve in systems with high penetration of wind power. In: Proceedings of the 7th Mediterranean conference and exhibition onpower generation, transmission, distribution and energy conversion (MedPower'10), Agia Napa, 7-10 Nov 2010, 9p

[21] Lobato Miguélez E, Egido Cortés I, Rouco Rodríguez L et al (2008) An overview of ancillary services in Spain. Electr Power Syst Res 78(3):515-523

[22] Ng WY (1981) Generalized generation distribution factors for power system security evaluations. IEEE Trans Power Appar Syst 100(3):1001-1005

[23] Biskas P, Bakirtzis A (2004) Decentralised security constrained DC-OPF of interconnected power systems. IEE P-Gener Transm Distrib 151(6):747-754

[24] Makarov YV, Loutan C, Ma J et al (2009) Operational impacts of wind generation on California power systems. IEEE Trans Power Syst 24(2):1039-1050

[25] Black M, Strbac G (2007) Value of bulk energy storage for managing wind power fluctuations. IEEE Trans Energ Conver 22(1):197-205

[26] Holttinen H, Milligan M, Kirby B et al (2008) Using standard deviation as a measure of increased operational reserve requirement for wind power. Wind Eng 32(4):355-377

[27] Grigg C, Wong P, Albrecht P et al (1999) The IEEE reliability test system-1996: A report prepared by the reliability test system task force of the application of probability methods subcommittee. IEEE Trans Power Syst 14(3):1010-1020

[28] Luo XC, Singh C, Patton AD (2003) Power system reliability evaluation using learning vector quantization and Monte Carlo simulation. Electr Power Syst Res 66(2):163-169

Jianxue WANG received the B.S., M.S. and Ph.D. degrees from $\mathrm{Xi}$ ' an Jiaotong University, Xi' an, China, all in electrical engineering, in 1999, 2002 and 2006, respectively. He is currently the Associate Professor in Power Systems Engineering at the Department of 
Electrical Engineering of Xi'an Jiaotong University from 2008. He worked in Brunel Institute of Power System of Brunel University, London, UK, as a Visiting Scientist from 2011 to 2012. His research fields include power system planning and operation, renewable energy, power markets and the design of energy management system of micro gird.

Ahmed Faheem ZOBAA received the B.Sc.(Hons.), M.Sc., and Ph.D degrees in electrical power and machines from Cairo University, Egypt, in 1992, 1997, and 2002, respectively. From 2007 to 2010, he was a Senior Lecturer in renewable energy at University of Exeter, U.K. He was also an Instructor from 1992 to 1997, a Teaching Assistant from 1997 to 2002, an Assistant Professor from 2003 to 2008, an Associate Professor from 2008 to 2013 at Cairo University where he has also been a Professor (on leave) since December 2013. Currently, he is a Senior Lecturer in power systems, an MSc Course Director and a Full Member of the Institute of Energy Futures at Brunel University London, U.K. His main areas of expertise are power quality, (marine) renewable energy, smart grids, energy efficiency, and lighting applications. He is an Editor-in-Chief for the International Journal of Renewable Energy Technology and Technology and Economics of Smart Grids and Sustainable Energy. $\mathrm{He}$ is also an Editorial Board member, Editor, Associate Editor, and Editorial Advisory Board member for many international journals. $\mathrm{He}$ is a registered Chartered Engineer, Chartered Energy Engineer,
European Engineer, and International Professional Engineer. He is also a registered member of the Engineering Council U.K., Egypt Syndicate of Engineers, and the Egyptian Society of Engineers. He is a Fellow of the Institution of Engineering and Technology, the Energy Institute of U.K., the Chartered Institution of Building Services Engineers, the Royal Society of Arts, the African Academy of Science, the Chartered Institute of Educational Assessors, and the Higher Education Academy of U.K. He is a senior member of the Institute of Electrical and Electronics Engineers. Also, $\mathrm{He}$ is a member of the International Solar Energy Society, the European Power Electronics and Drives Association, the British Institute of Energy Economics, and the IEEE Standards Association.

Chengchen HUANG received the B.S. and M.S. degrees from Xi'an Jiaotong University, Xi'an, China, all in electrical engineering, in 2010 and 2013, respectively. His research fields include the power system planning and operation.

Chen CHEN received the B.S. degree from Northeast Dianli University, Jilin, China, in electrical engineering, in 2007, and received the M.S. degree from Xi'an Jiaotong University, Xi'an, China, in electrical engineering, in 2010. Her research fields include renewable energy and power system operation. 